Canadian

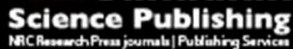

Applied Physiology, Nutrition, and Metabolism Physiologie appliquée, nutrition et métabolisme

\title{
Examination of associations between chaos in the home environment, serum cortisol level, and dietary fat intake among parents of preschool-age children
}

\begin{tabular}{|r|l|}
\hline Journal: & Applied Physiology, Nutrition, and Metabolism \\
\hline Manuscript ID & apnm-2016-0659.R1 \\
\hline Manuscript Type: & Brief communication \\
\hline Complete List of Authors: & $\begin{array}{l}\text { MacRae, Laura; University of Guelph, Human Health and Nutritional } \\
\text { Sciences } \\
\text { Darlington, Gerarda; University of Guelph, Dept of Mathematics and } \\
\text { Statistics } \\
\text { Haines, Jess; University of Guelph, Family Relations and Applied Nutrition } \\
\text { Ma, David; University of Guelph, }\end{array}$ \\
\hline Keyword: & Chaos, Cortisol, diet < diet, Parent, stress < stress \\
\hline \multicolumn{2}{|c}{} \\
\hline
\end{tabular}

SCHOLARONE

Manuscripts 


\section{Examination of associations between chaos in the home environment, serum cortisol level,} and dietary fat intake among parents of preschool-age children

Laura M. MacRae ${ }^{\mathrm{a}}$, Gerarda Darlington ${ }^{\mathrm{b}}$, Jess Haines ${ }^{\mathrm{c}^{* *}}$, David W.L. Ma ${ }^{\mathrm{a}^{*}}$, on behalf of the Guelph Family Health Study

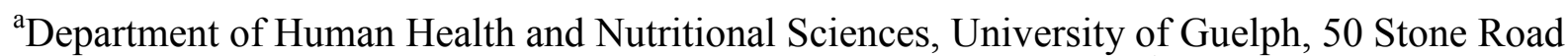
East, Guelph, ON, N1G 2W1,Canada; e-mail: macrael@uoguelph.ca ${ }^{1}$

${ }^{b}$ Department of Mathematics and Statistics, University of Guelph, 50 Stone Road East, Guelph, ON, N1G 2W1,Canada; e-mail: gdarling@uoguelph.ca

${ }^{\mathrm{c}}$ Department of Family Relations and Applied Nutrition, University of Guelph, 50 Stone Road East, Guelph, ON, N1G 2W1,Canada; e-mail: jhaines@uoguelph.ca

*Co-corresponding author at: Department of Human Health and Nutritional Sciences, University of Guelph, 50 Stone Road East, Guelph, ON, N1G 2W1,Canada e-mail address: davidma@uoguelph.ca

**Co-corresponding author at: Department of Family Relations and Applied Nutrition, University of Guelph, 50 Stone Road East, Guelph, ON, N1G 2W1,Canada

e-mail address: jhaines@uoguelph.ca

Abbreviations: CHAOS (Confusion, Hubbub, and Order Scale); HPA (Hypothalamic-PituitaryAdrenal Axis); BMI (Body Mass Index); SD (Standard Deviation); CI (Confidence Interval) 


\begin{abstract}
This research investigated associations between home environment chaos, fat intake, and morning serum cortisol level in 44 parents of preschool-age children. Chaos was measured using the Confusion, Hubbub, and Order Scale, and fat intake was quantified using the Block fat screener. Linear regression analyses demonstrated that chaos and cortisol were both associated with fat intake $(\hat{\beta}=0.53, p=0.001 ; \hat{\beta}=0.03, p=0.0002)$, and there was a non-significant association between chaos and cortisol.

Keywords: Chaos; Cortisol; Diet; Parent; Stress
\end{abstract}




\section{Introduction}

Nearly two thirds of Canadian adults and one third of Canadian children are overweight or obese (SOCI, 2016). To develop effective interventions for obesity, it is important to identify key modifiable risk factors. One modifiable factor that has gained interest in recent years is the influence of stress on dietary intake.

Stress can contribute to the development of obesity by promoting unhealthy dietary patterns and eating behaviours (Sominsky \& Spencer, 2014). A stressful situation activates the hypothalamic-pituitary-adrenal (HPA) axis, which results in an increased production of cortisol (Spencer \& Tilbrook, 2011). Cortisol is known to stimulate appetite and enhance the rewarding nature of foods that are high in sugar and fat content, which in chronic stress situations can promote weight gain (Sominsky \& Spencer, 2014).

Evidence supporting the need for obesity prevention programs that include stress management is increasing (Campbell, 2015). However, influences of particular types of chronic stress on dietary intake have rarely been investigated. The Confusion, Hubbub, and Order Scale (CHAOS) is a validated measure of noise, crowding, and routines in the family home environment (Matheny et al., 1995). Two studies have demonstrated positive associations between home environment chaos, as measured using the CHAOS survey, and obesity-related behaviours, including poor sleep and emotional overeating in children (Boles et al., 2016; Lumeng et al., 2014). It is not known, however, what effect chaos may have on behaviours that increase obesity risk in parents. This is important to investigate, because children's eating habits are learned from their parents and are established early in life (Patrick \& Nicklas, 2005; Savage, Fisher, \& Birch, 2007). If parents are modeling stress-related eating habits, these behaviours may be passed on to their children. It has been demonstrated that fat intake correlates with BMI 
(Hooper et al. 2015). Therefore, the objective of this research was to examine associations between home environment chaos, serum cortisol levels, and dietary fat intake among parents of the Guelph Family Health Study, a family-based cohort study. The primary hypothesis was that home environment chaos would be positively associated with fat intake, and the secondary hypothesis was that cortisol would have a mediating influence on this relationship.

\section{Methods}

Participants

There were 79 parents from 44 families who participated in the pilot phase of the Guelph Family Health Study. Inclusion criteria were families who had at least one child between the ages of 18 months and 5 years, and who lived in the Guelph-Wellington area. Baseline data for home environment chaos, cortisol, and fat intake were available for 45 parents from 30 families. One participant's cortisol value was considered an outlier, as it was greater than three standard deviations above the mean. Data from this participant were not included in the current analyses. Excluding participants who were non-fasted for their blood sample collection did not yield substantively different results, and therefore both fasted and non-fasted participants were included. The final sample size was 44 parents from 30 families, with 10 of those parents being non-fasted participants. The study was approved by the University of Guelph Research Ethics Board (RCT 02223234). Parents provided written informed consent to participate in this research.

CHAOS

One parent from each family completed the survey to provide a CHAOS score for their household (Matheny et al., 1995). This 15-item measure assesses the presence of noise, clutter, and routines within the home. Participants ranked, on a scale of 1 to 4 , how strongly they agreed 
that the statement described their home. A total CHAOS score was then calculated for each family.

Diet

Parents' fat intake was quantified using the Block fat screener (Block, Gillespie, Rosenbaum, \& Jenson, 2000). Participants ranked on a scale of 1 (once per month or less) to 5 (5 or more times per week) how often they consumed 16 high-fat food items. A total fat screener score was calculated for each participant by summing the responses.

\section{Cortisol}

Blood samples were collected from participants in the morning by Lifelabs Medical Laboratory Services in Guelph. Serum cortisol levels were also analyzed by Lifelabs using the Siemens Advia Centaur cortisol assay, a competitive immunoassay with chemiluminescent detection. Lifelabs did not require participants to fast for this test.

\section{Data for covariate analyses}

Data for participant sex, household income, and family size were collected through participant self-report. Participants reported their total annual household income on a scale of 1 to 12 , with 1 being less than $\$ 10,000$ and 12 being $\$ 150,000$ or more. Income category midpoints for levels 1 to 11 and minimum for level 12 were used to create a quantitative income variable. Family size was a three level categorical variable defined as either three, four, or more than four people living in the household.

\section{Statistical Analyses}

Data analysis was performed using SAS version 9.2. Linear regression analyses were performed to investigate associations among dietary fat, home environment chaos, and cortisol. Robust standard errors were computed via the use of generalized estimating equations in order to 
account for dependent observations within families (Zeger \& Liang, 1986). First, the association between home environment chaos and fat intake was investigated. Second, a mediating effect of cortisol was investigated by examining associations between home environment chaos and cortisol, and between cortisol and fat intake. Cortisol was also investigated as a covariate in a model examining the association between home environment chaos and fat intake. Adjusted models included participant sex, household income, and family size. A significance level of 0.05 was used.

\section{Results}

\section{Participant Demographics}

The participant demographics are outlined in Table 1. There were 23 females and 21 males, with a mean age of 34.6 years. The BMI scores of the participants ranged from normal weight $\left(19.0 \mathrm{~kg} / \mathrm{m}^{2}\right)$ to obese $\left(47.2 \mathrm{~kg} / \mathrm{m}^{2}\right)$, with a mean BMI of $28.4 \mathrm{~kg} / \mathrm{m}^{2}$. There was a wide income distribution amongst the families $(\$ 30,000$ to $\geq \$ 150,000)$, and the median household income was $\$ 90,000$. A majority $(84 \%)$ of the participants were White, with the remainder of the participants identifying as South Asian, Chinese, and Latin American.

\section{Linear Regression Analysis Results}

Associations between chaos in the home environment, dietary fat, and cortisol are presented in Table 2. Significant positive associations were observed between home environment chaos and parents' fat intake $(\hat{\beta}=0.53(\mathrm{CI}=0.21-0.85), \mathrm{p}=0.001)$, as well as between parents' cortisol levels and fat intake $(\hat{\beta}=0.03(\mathrm{CI}=0.02-0.05), \mathrm{p}=0.0002)$, based on models that adjusted for participant sex, household income, and family size. Over $80 \%$ of the families were twoparent families, and so models that adjusted for number of children in the household instead of family size were also run. These models yielded similar results (results not shown). There was a 
non-significant positive association observed between home environment chaos and parents' cortisol levels $(\hat{\beta}=2.86(\mathrm{CI}=-1.92-7.65), \mathrm{p}=0.24)$.

Including cortisol in the adjusted model of the association between home environment chaos and fat intake resulted in a decrease in the coefficient estimate for chaos from 0.53 to 0.44 $(\mathrm{CI}=0.11-0.77)$. The association between home environment chaos and fat intake remained significant after this adjustment $(\mathrm{p}=0.01)$.

\section{Discussion}

The results supported our primary hypothesis that home environment chaos would be positively associated with parents' fat intake. This is the first study to investigate the association between home environment chaos and parents' dietary intake. Past research has demonstrated that parents have an important influence on the eating habits that their children develop (Patrick \& Nicklas, 2005; Savage et al., 2007). Therefore, if parents from more chaotic homes are modelling high-fat eating habits, this could have a negative influence on their own health as well as the health of their children.

Previous research has been inconclusive with regards to how the association between stress and dietary intake may differ by sex (O’Connor et al. 2008, Tan and Chow 2014). The results of the current research demonstrated that the association between home environment chaos and fat intake was independent of participant sex, suggesting that the dietary intake of both males and females can be influenced by home environment chaos.

Individuals living in low-income households tend to be at a greater risk for chronic stress and poor quality diets (Darmon and Drewnowski 2008). However, our results indicated that home environment chaos was associated with parents' fat intake independent of household 
income. Furthermore, home environment chaos was associated with parents' fat intake independent of family size. Thus, our results suggest that families of varying size and socioeconomic status would benefit from interventions designed to reduce the level of chaos within the home.

Our results showed that parents with higher serum cortisol levels were more likely to have a diet high in fat, and this was true independent of participant sex, household income, and family size. This finding is consistent with the hypothesized role of cortisol in promoting intake of energy-dense foods. The proposed mechanism for this response involves cortisol's interaction with appetite-stimulating and reward-enhancing areas of the brain (Sominsky \& Spencer, 2014).

Adding cortisol to the model of the association between home environment chaos and fat intake resulted in a slight decrease in the coefficient estimate for chaos. This lends support to the hypothesis that participants' cortisol levels may have an influence on the relationship between home environment chaos and fat intake. However, due to the lack of a significant association between home environment chaos and cortisol, we did not demonstrate a mediating influence of cortisol on the relationship between home environment chaos and fat intake.

While it is possible that home environment chaos and cortisol were each independently associated with parents' fat intake, it is also possible that our non-significant finding between home environment chaos and cortisol is due to the fact that our measure of cortisol may not be an accurate reflection of participants' chronic stress levels. Serum cortisol only measures the level of circulating cortisol at the point of sampling. Circulating cortisol levels can be influenced by factors such as recent exercise (Hill et al. 2008), sleep quality (Gonnissen et al. 2013), and acute stress (Foley and Kirschbaum 2010). We did not collect data on these variables at the time of blood collection, which is a limitation of our study design. An interesting future direction for this 
research would be to analyze cortisol concentrations in scalp hair samples, which have been demonstrated to reflect participants' average exposure to cortisol over a period of months (Russell et al., 2012), and explore the potential mediating influence of cortisol on the association between home environment chaos and fat intake.

Strengths of this research include the use of both male and female participants, inclusion of a physiological and a subjective measure of stress, and the novelty of investigating an association between home environment chaos and dietary intake in parents of preschool-age children. There are also limitations, including a small sample size, the use of serum cortisol rather than a chronic measure of physiological stress, and a lack of data available on other aspects of the parents' diets. Our measure of fat intake was based on frequencies, not on quantity of fat consumed. However, the Block fat screener is a validated survey that has been shown to correlate with fat intake data collected from four-day food diaries (Block et al. 1989). Finally, in this study only one parent provided information on the level of chaos in the home. It is possible that, in two-parent homes, the parents have different interpretations of how chaotic their home is, which could influence their response to the level of home environment chaos. Future research should consider the use of hair cortisol analysis, assessment of home environment chaos from both parents in two-parent homes, and the collection of more complete dietary data.

\section{Conclusion}

Our results demonstrated a significant positive association between home environment chaos and fat intake among parents of preschool-age children after adjusting for covariates. However, our secondary hypothesis that cortisol would have a mediating influence on this relationship was not supported. While additional research is needed to elucidate the influence of chaos in the home environment on stress and dietary intake, these results suggest that chaos in 
the home may be an important consideration when exploring dietary risk factors for obesity among families with preschool-age children.

\section{Conflict of Interest}

The authors report no conflicts of interest associated with this manuscript.

\section{Acknowledgements}

Study Coordinator: Angela Annis

Funding: This work was supported by the Health for Life Initiative at the University of Guelph [grant number NA] 


\section{References}

Block, G., Clifford, C., Naughton, M.D., Henderson, M., and McAdams, M. 1989. A brief dietary screen for high fat intake. J. Nutr. Educ. 21(5): 199-207. Society for Nutrition Education and Behavior, doi:http://dx.doi.org/10.1016/S0022-3182(89)80003-2.

Block, G., Gillespie, C., Rosenbaum, E.H., and Jenson, C. 2000. A rapid food screener to assess fat and fruit and vegetable intake. Am. J. Prev. Med. 18(4): 284-288. doi:10.1016/S07493797(00)00119-7.

Boles, R.E., Halbower, A.C., Daniels, S., Gunnarsdottir, T., Whitesell, N., and Johnson, S.L. 2016. Family chaos and child functioning in relation to sleep problems among children at risk for obesity. Behav. Sleep Med.: 1-15. doi:10.1080/15402002.2015.1104687.

Campbell, M.K. 2015. Biological, environmental and social influences on childhood obesity. Pediatr. Res. 79: 1-7. doi:10.1038/pr.2015.208.

Darmon, N., and Drewnowski, A. 2008. Does social class predict diet quality? Am. J. Clin. Nutr. 87(5) doi:87/5/1107 [pii].

Foley, P., and Kirschbaum, C. 2010. Human hypothalamus-pituitary-adrenal axis responses to acute psychosocial stress in laboratory settings. Neurosci. Biobehav. Rev. 35(1): 91-96. doi:10.1016/j.neubiorev.2010.01.010.

Gonnissen, H.K.J., Hursel, R., Rutters, F., Martens, E.A.P., and Westerterp-Plantenga, M.S. 2013. Effects of sleep fragmentation on appetite and related hormone concentrations over $24 \mathrm{~h}$ in healthy men. Br. J. Nutr. 109(4): 748-756. doi:10.1017/S0007114512001894.

Hill EE, Zac E, Battaglini C, Viru M, Viru A, H.A. 2008. Exercise and circulating cortisol levels: the inensity theshold effect. J Endocrinol Invest. 31(7): 587-591. doi:4732 [pii].

Hooper, L., Abdelhamid, A., Bunn, D., Brown, T., Cd, S., and Cm, S. 2015. Effects of total fat 
intake on body weight. The Cochrane Library, (8).

doi:10.1002/14651858.CD011834.www.cochranelibrary.com.

Lumeng, J.C., Miller, A., Peterson, K.E., Kaciroti, N., Sturza, J., Rosenblum, K., and Vazquez, D.M. 2014. Diurnal cortisol pattern, eating behaviors and overweight in low-income preschool-aged children. Appetite, 73: 65-72. doi:10.1016/j.appet.2013.10.016.

Matheny, A.P., Wachs, T.D., Ludwig, J.L., and Phillips, K. 1995. Bringing order out of chaos: Psychometric characteristics of the confusion, hubbub, and order scale. J. Appl. Dev. Psychol. 16(3): 429-444. doi:10.1016/0193-3973(95)90028-4.

O’Connor, D.B., Jones, F., Conner, M., McMillan, B., and Ferguson, E. 2008. Effects of daily hassles and eating style on eating behavior. Heal. Psychol. 27(1 Suppl): S20-S31. doi:10.1037/0278-6133.27.1.S20.

Patrick, H., and Nicklas, T.A. 2005. A review of family and social determinants of children's eating patterns and diet quality. J. Am. Coll. Nutr. 24(2): 83-92. doi:10.1080/07315724.2005.1071944.

Russell, E., Koren, G., Rieder, M., and Uum, S. Van. 2012. Hair cortisol as a biological marker of chronic stress: Current status, future directions and unanswered questions. Psychoneuroendocrinology, 37(5): 589-601. doi:10.1016/j.psyneuen.2011.09.009.

Savage, J.S., Fisher, J.O., and Birch, L.L. 2007. Parental influence on eating behavior: conception to adolescence. J. Law Med. Ethics, 35(1): 22-34. doi:doi: 10.1111/j.1748720X.2007.00111.x.

SOCI. 2016. Obesity in Canada: A whole-of-society approach for a healthier Canada. Available from http://www.parl.gc.ca/content/sen/committee/421/SOCI/Reports/2016-0225_Revised_report_Obesity_in_Canada_e.pdf [accessed 20 June 2016]. 
Sominsky, L., and Spencer, S.J. 2014. Eating behavior and stress: a pathway to obesity. Front. Psychol. 5: 1-8. doi:10.3389/fpsyg.2014.00434.

Spencer, S.J., and Tilbrook, A. 2011. The glucocorticoid contribution to obesity. Stress, 14(3): 233-46. doi:10.3109/10253890.2010.534831.

Tan, C.C., and Chow, C.M. 2014. Stress and emotional eating: The mediating role of eating dysregulation. Pers. Individ. Dif. 66: 1-4. doi:10.1016/j.paid.2014.02.033.

Zeger, S.L., and Liang, K.Y. 1986. Longitudinal data analysis for discrete and continuous outcomes. Biometrics, 42(1): 121-130. doi:10.2307/2531248. 
Table 1. Characteristics of parents $(n=44)$ from 30 families in the Guelph Family Health Study

\begin{tabular}{|l|l|}
\hline Variable & n (\%), or mean (SD) \\
\hline Sex & \\
\hline Male & $21(48 \%)$ \\
\hline Female & $23(52 \%)$ \\
\hline Age (years) mean (SD) & $34.6(3.3)$ \\
\hline BMI (kg/m $\left.{ }^{2}\right)$ & \\
\hline Normal weight (18.5-24.9) & $20(45 \%)$ \\
\hline \multicolumn{1}{|c|}{ Overweight $(25-29.9)$} & $10(23 \%)$ \\
\hline \multicolumn{1}{|c|}{ Obese $(\geq 30)$} & $14(32 \%)$ \\
\hline Household Income & $6(14 \%)$ \\
\hline \$30,000-\$49,999 & $9(20 \%)$ \\
\hline \$50,000-\$69,999 & $10(23 \%)$ \\
\hline \$70,000-\$99,999 & $19(43 \%)$ \\
\hline$\geq \$ 100,000$ & $8(27 \%)$ \\
\hline Family Size & $17(57 \%)$ \\
\hline Families with 3 members & $5(16 \%)$ \\
\hline Families with 4 members & $37(84 \%)$ \\
\hline Families with $>4$ members & $6(14 \%)$ \\
\hline Ethnicity & $1(2 \%)$ \\
\hline White & $38.4(7.4)$ \\
\hline Other & $31.1(6.2)$ \\
\hline No response & $300.0(104.4)$ \\
\hline $\begin{array}{l}\text { Fat Screener Score[range: } \mathbf{1 6 - 8 0 ]} \\
\text { mean (SD) }\end{array}$ & \\
\hline $\begin{array}{l}\text { CHAOS Score [range: 15-60] } \\
\text { mean (SD) }\end{array}$ & \\
\hline Cortisol (nmol/L) mean (SD) & \\
\hline
\end{tabular}


Table 2. Unadjusted and adjusted associations among fat screener score, CHAOS score, and cortisol

\begin{tabular}{|l|l|l|l|l|l|l|}
\hline \multirow{2}{*}{ Association } & \multicolumn{3}{|c|}{ Unadjusted } & \multicolumn{3}{c|}{$\begin{array}{c}\text { Adjusted for sex, income, and } \\
\text { family size }\end{array}$} \\
\cline { 2 - 7 } & $\begin{array}{l}\text { Unstandardized } \\
\text { Coefficient } \\
\text { Estimate }\end{array}$ & $\begin{array}{l}95 \% \\
\text { Confidence } \\
\text { Interval }\end{array}$ & $p$-value & $\begin{array}{l}\text { Unstandardized } \\
\text { Coefficient } \\
\text { Estimate }\end{array}$ & $\begin{array}{l}95 \% \\
\text { Confidence } \\
\text { Interval }\end{array}$ & -value \\
\hline $\begin{array}{l}\text { Fat Intake } \\
\text { vs. CHAOS }\end{array}$ & 0.43 & $0.23-0.64$ & $<\mathbf{0 . 0 0 0 1}$ & 0.53 & $0.21-0.85$ & $\mathbf{0 . 0 0 1}$ \\
\hline $\begin{array}{l}\text { Fat Intake } \\
\text { vs. Cortisol }\end{array}$ & 0.03 & $0.01-0.05$ & $\mathbf{0 . 0 0 2}$ & 0.03 & $0.02-0.05$ & $\mathbf{0 . 0 0 0 2}$ \\
\hline $\begin{array}{l}\text { Cortisol vs. } \\
\text { CHAOS }\end{array}$ & 2.67 & $-1.75-7.10$ & 0.24 & 2.86 & $-1.92-7.65$ & 0.24 \\
\hline
\end{tabular}

